\title{
SUBDIFFERENTIAL CALCULUS RULES FOR SUPREMUM FUNCTIONS IN CONVEX ANALYSIS*
}

\author{
CHONG $\mathrm{LI}^{\dagger}$ AND K. F. NG ${ }^{\ddagger}$
}

\begin{abstract}
Extending and improving some recent results of Hantoute, López, and Zălinescu and others, we provide characterization conditions for subdifferential formulas to hold for the supremum function of a family of convex functions on a real locally convex space.
\end{abstract}

Key words. convex analysis, supremum function, convex subdifferential

AMS subject classifications. 52A41, 90C25, 15A39

DOI. $10.1137 / 100808344$

1. Introduction. Let $X$ be a real locally convex (Hausdorff topological vector) space. Consider the family $\left\{f_{i}: i \in I\right\}$ of proper convex functions on $X$, where $I$ is a (finite or infinite) index set. Let $\psi$ denote the (pointwise) supremum function of $\left\{f_{i}: i \in I\right\}$, defined by

$$
\psi(x):=\sup \left\{f_{i}(x): i \in I\right\} \quad \text { for each } x \in X .
$$

Supremum functions arise in a variety of contexts, including dualities, extended Farkas lemmas, semi-infinite programming, and convex optimization; see, for example, [3, 4, 5, $6,7,8,12,13,14,15,16,18,19,20,21,25,29]$. Calculus for subdifferentials of supremum functions is one of the most important issues and plays a crucial role in convex analysis and variational problems; it has been studied extensively and deeply under various degrees of generality (such as that $X$ is finite dimensional, $I$ is finite, or the data functions $f_{i}$ are continuous/lower semicontinuous) by many authors, including Brøndsted [2], Levin [17], Valadier [26], Ioffe and Tikhomirov [15], and others [10, 12, 20, 22, 23, $24,25,27,28,29]$. The key problem considered by these authors in this connection is to represent the subdifferential $\partial \psi(z)$ of the supremum function $\psi$ at $z \in \operatorname{dom} \psi$ in terms of the subdifferentials/ $\epsilon$-subdiffererntials of the data functions $f_{i}$ at $z$. Extending all the earlier works regarding this problem, Hantoute, López, and Zălinescu showed recently in [11] that, for the most general situation $(X$ is a real locally convex space and the functions $f_{i}$ are not necessarily lower semicontinuous (lsc)), if the lsc hulls of $\psi$ and $f_{i}$ satisfy the condition

$$
\operatorname{cl} \psi(x)=\sup _{i \in I} \operatorname{cl} f_{i}(x) \quad \text { for each } x \in X,
$$

then

*Received by the editors September 13, 2010; accepted for publication (in revised form) June 1, 2011; published electronically August 25, 2011.

http://www.siam.org/journals/siopt/21-3/80834.html

'Department of Mathematics, Zhejiang University, Hangzhou 310027, People's Republic of China and Department of Mathematics, College of Sciences, King Saud University, P. O. Box 2455, Riyadh 11451, Saudi Arabia (cli@zju.edu.cn). The first author was supported in part by the National Natural Science Foundation of China (grant 10731060) and by Zhejiang Provincial Natural Science Foundation of China (grant Y6110006).

Department of Mathematics (and IMS), Chinese University of Hong Kong, Hong Kong, People’s Republic of China (kfng@math.cuhk.edu.hk). The second author was supported by a General Research Fund (GRF) grant from the Research Grant Council of Hong Kong. 


$$
\partial \psi(z)=\bigcap_{L \in \mathcal{F}_{z}, \epsilon>0} \operatorname{cl}\left(\operatorname{co}\left(\cup_{i \in I_{\epsilon}(z)} \partial_{\epsilon} f_{i}(z)\right)+\mathrm{N}_{L \cap \operatorname{dom} \psi}(z)\right) \quad \text { for each } z \in X,
$$

where $I_{\epsilon}(z):=\left\{i \in I: f_{i}(z) \geq \psi(z)-\epsilon\right\}$ and $\mathcal{F}_{z}$ denotes the set of all finite-dimensional subspaces $L$ of $X$ containing $z$; in particular, (1.2) entails

$$
\partial \psi(z)=\bigcap_{\epsilon>0} \operatorname{cl}\left(\operatorname{co}\left(\cup_{i \in I_{\epsilon}(z)} \partial_{\epsilon} f_{i}(z)\right)+\mathrm{N}_{\operatorname{dom} \psi}(z)\right) \quad \text { for each } z \in X,
$$

when $X$ is a Euclidean space. The formulas such as (1.3) have been established in [9] and $[10]$ in the finite-dimensional space.

The main result of this paper reported in section 3 implies that, for the same conclusion of [11] mentioned above, (1.1) can be replaced by the following condition:

$$
\operatorname{cl} \psi(x)=\sup _{i \in I} \operatorname{cl} f_{i}(x) \quad \text { for each } x \in \bigcup_{L \in \mathcal{F}} \operatorname{cl}(L \cap \operatorname{dom} \psi),
$$

where $\mathcal{F}$ is the set of all finite-dimensional subspaces; this new condition is strictly weaker than (1.1) even when $I$ is finite and $X$ is finite dimensional (see Example 3.2). We show, in fact, that (1.4) and (1.2) are equivalent if $X$ is a Banach space.

The rest of the paper is organized as follows. In section 2 , we list some basic concepts and notations; we also prove several preliminary results on normal cones, epigraphs, and supremum functions as well as the relationships between them. These results are needed in section 3, where the main results and some examples are presented.

2. Notations and preliminary results. The notation used in the present paper is standard (cf. $[12,29])$. In particular, we assume throughout the whole paper (unless otherwise specified) that $X$ is a real locally convex Hausdorff topological vector space and let $X^{*}$ denote the dual space of $X$ equipped with the weak*-topology, whereas $\left\langle x^{*}, x\right\rangle$ denotes the value of a functional $x^{*}$ in $X^{*}$ at $x \in X$, i.e., $\left\langle x^{*}, x\right\rangle=x^{*}(x)$. Let $Z$ be a set in $X\left(\right.$ or $\left.X^{*}\right)$. The interior (respectively, closure, convex hull, convex cone hull, linear hull, affine hull) of $Z$ is denoted by int $Z$ (respectively, $\operatorname{cl} Z, \operatorname{co} Z$, cone $Z, \operatorname{span} Z$, aff $Z$ ). Following [29], the relative interior of $Z$ is denoted by ri $Z$ and defined to be the interior of $Z$ in the topology relative to aff $Z$ if aff $Z$ is closed and the empty otherwise. Then, by definition, one can easily prove that a point $x \in \operatorname{ri} Z$ if and only if there exists $\delta>0$ such that $\mathbf{B}(x, \delta) \cap \overline{\operatorname{aff} Z} \subseteq Z$. Furthermore, for convex $Z$, we have that $\operatorname{ri} Z=\operatorname{ri}(\operatorname{cl} Z)$ and so (cf. [1, Lemma 3.1])

$$
\lambda x+(1-\lambda) y \in \operatorname{ri}(\operatorname{cl} Z)=\operatorname{ri} Z \quad \text { for any } x \in \operatorname{ri} Z, \quad y \in \operatorname{cl} Z, \quad \text { and } \quad \lambda \in(0,1] .
$$

The negative polar cone $Z^{\ominus}$ of $Z$ is defined by

$$
Z^{\ominus}:=\left\{x^{*} \in X^{*}:\left\langle x^{*}, z\right\rangle \leq 0 \text { for all } z \in Z\right\} .
$$

The normal cone of $Z$ at $z_{0} \in Z$ is denoted by $\mathrm{N}_{Z}\left(z_{0}\right)$ and is defined by $\mathrm{N}_{Z}\left(z_{0}\right)=$ $\left(Z-z_{0}\right)^{\ominus}$. The indicator function $\delta_{Z}$ and the support function $\sigma_{Z}$ of $Z$ are, respectively, defined by

$$
\delta_{Z}(x):=\left\{\begin{array}{cc}
0 & x \in Z \\
\infty & \text { otherwise }
\end{array}\right.
$$

and

Copyright (c) by SIAM. Unauthorized reproduction of this article is prohibited. 


$$
\sigma_{Z}\left(x^{*}\right):=\sup _{x \in Z}\left\langle x^{*}, x\right\rangle \quad \text { for each } x^{*} \in X^{*} .
$$

Let $f: X \rightarrow \overline{\mathbb{R}}:=\mathbb{R} \cup\{+\infty\}$ be a proper function. Let $f^{*}$ denote its conjugate function, that is,

$$
f^{*}\left(x^{*}\right):=\sup \left\{\left\langle x^{*}, x\right\rangle-f(x): x \in X\right\} \quad \text { for each } x^{*} \in X^{*} .
$$

The epigraph of a function $f$ on $X$ is denoted by epi $f$ and defined by

$$
\text { epi } f:=\{(x, r) \in X \times \mathbb{R}: f(x) \leq r\} .
$$

It is easy to see that, for two proper functions $f$ and $g$,

$$
f \leq g \Rightarrow f^{*} \geq g^{*} \Leftrightarrow \operatorname{epi} f^{*} \subseteq \operatorname{epi} g^{*} .
$$

The closure of $f$ is denoted by $\operatorname{cl} f$, which is defined by

$$
\operatorname{epi}(\operatorname{cl} f)=\operatorname{cl}(\operatorname{epi} f) \text {. }
$$

Then (cf. [29, Theorems 2.3.1]),

$$
f^{*}=(\operatorname{cl} f)^{*} .
$$

For a proper convex function $f$, the subdifferential $\partial f(x)$ of $f$ at $x \in X$ is defined by

$$
\partial f(x):=\left\{x^{*} \in X^{*}: f(x)+\left\langle x^{*}, y-x\right\rangle \leq f(y) \text { for each } y \in X\right\},
$$

and, for $\epsilon>0$, one defines the $\epsilon$-subdifferential $\partial_{\epsilon} f(x)$ of $f$ at $x \in X$ by

$$
\partial_{\epsilon} f(x):=\left\{x^{*} \in X^{*}: f(x)+\left\langle x^{*}, y-x\right\rangle \leq f(y)+\epsilon \text { for each } y \in X\right\} .
$$

As is well known, the Young-Fenchel inequality below holds:

$$
f(x)+f^{*}\left(x^{*}\right) \geq\left\langle x^{*}, x\right\rangle \quad \text { for each pair }\left(x^{*}, x\right) \in X \times X^{*} .
$$

Moreover, by [29, Theorem 2.4.2 (iii)],

$$
f(x)+f^{*}\left(x^{*}\right)=\left\langle x^{*}, x\right\rangle \quad \text { if and only if } x^{*} \in \partial f(x)
$$

(the equality in (2.5) is usually referred to as Young's equality). In particular,

$$
\left(x^{*},\left\langle x^{*}, x\right\rangle-f(x)\right) \in \operatorname{epi} f^{*} \quad \text { for each } x^{*} \in \partial f(x),
$$

and the following statements hold for a convex subset $Z$ of $X$ :

$$
\sigma_{Z}=\delta_{Z}^{*}, \quad \mathrm{~N}_{Z}(x)=\partial \delta_{Z}(x) \quad \text { for each } x \in Z .
$$

The following lemma is well known; see, for example, [29, Theorem 2.8.7].

Lemma 2.1. Let $g, h: X \rightarrow \overline{\mathbb{R}}$ be convex lower semicontinuous functions satisfying $\operatorname{dom} g \cap \operatorname{dom} h \neq \varnothing$. Then the following assertion regarding epigraphs holds:

$$
\operatorname{epi}(g+h)^{*}=\operatorname{cl}\left(\operatorname{epi} g^{*}+\operatorname{epi} h^{*}\right) .
$$

Copyright ( $\odot$ by SIAM. Unauthorized reproduction of this article is prohibited. 
2.1. Normal cones and epigraphs. We begin this subsection with the following lemma.

Lemma 2.2. Consider convex sets $E$ and $B$ in $X$. Suppose that $E$ is closed and $E \cap$ ri $B \neq \varnothing$. Let $x \in E \cap B$. Then we have that

$$
\text { epi } \sigma_{E \cap B}=\operatorname{cl}\left(\text { epi } \sigma_{E}+\text { epi } \sigma_{B}\right) \text {. }
$$

If $E$ is additionally affine, we have that

$$
\mathrm{N}_{E \cap B}(x)=\operatorname{cl}\left(\mathrm{N}_{E}(x)+\mathrm{N}_{B}(x)\right) .
$$

Proof. We note by assumption that $\operatorname{cl}[E \cap B]=E \cap \operatorname{cl} B$ and so epi $\sigma_{E \cap B}=$ epi $\delta_{E \cap(\mathrm{cl} B)}^{*}($ see $(2.7))$. It follows from Lemma 2.1 that

$$
\operatorname{epi} \sigma_{E \cap B}=\operatorname{epi}\left(\delta_{E}+\delta_{\mathrm{cl} B}\right)^{*}=\operatorname{cl}\left(\operatorname{epi} \delta_{E}^{*}+\operatorname{epi} \delta_{\mathrm{cl} B}^{*}\right)=\operatorname{cl}\left(\operatorname{epi} \sigma_{E}+\operatorname{epi} \sigma_{B}\right)
$$

and (2.9) is shown.

Now assume additionally that $E$ is affine. To show (2.10), we have by the assumption that $(E-x) \cap \operatorname{ri}(B-x) \neq \varnothing$. This implies that $(E-x) \cap \operatorname{ri}[\operatorname{cone}(B-x)] \neq \varnothing$. Thus, using (2.1), one easily sees that

$$
\operatorname{cl}[(E-x) \cap \operatorname{cone}(B-x)]=(E-x) \cap \operatorname{cl}[\operatorname{cone}(B-x)] .
$$

Moreover, it is clear that cone $[(E \cap B)-x]=(E-x) \cap \operatorname{cone}(B-x)$ and so

$$
\operatorname{cl}[\operatorname{cone}((E \cap B)-x)]=(E-x) \cap \operatorname{cl}[\operatorname{cone}(B-x)] .
$$

We apply the bipolar theorem to conclude that

$$
\begin{aligned}
\mathrm{N}_{E \cap B}(x) & =((E-x) \cap \operatorname{cl}[\operatorname{cone}(B-x)])^{\ominus} \\
& =\operatorname{cl}\left[\operatorname{co}\left((E-x)^{\ominus} \cup(\operatorname{cl}[\operatorname{cone}(B-x)])^{\ominus}\right)\right] \\
& =\operatorname{cl}\left(\mathrm{N}_{E}(x)+\mathrm{N}_{B}(x)\right)
\end{aligned}
$$

and (2.10) is seen to hold.

For any convex subset $D$ of $X$, we use $\tilde{\mathcal{F}}_{D}$ to denote the family of all subspaces $L$ of $X$, satisfying the property that $\operatorname{ri}(L \cap D) \neq \varnothing$. Let $\mathcal{F}_{D}$ denote the subfamily of $\tilde{\mathcal{F}}_{D}$ consisting of all finite-dimensional subspaces $L \in \tilde{\mathcal{F}}_{D}$. The families $\tilde{\mathcal{F}}_{\text {dom } \psi}$ and $\mathcal{F}_{\text {dom } \psi}$ will be denoted by $\tilde{\mathcal{F}}_{\psi}$ and $\mathcal{F}_{\psi}$ for simplicity. Similar understanding is for $\tilde{\mathcal{F}}_{\mathrm{cl} \psi}$ and $\mathcal{F}_{\mathrm{cl} \psi}$. For $x \in D, \mathcal{F}_{x, D}$ stands for the subfamily of $\mathcal{F}_{D}$ consisting of all $L$ in $\mathcal{F}_{D}$ containing $x$. Similar understanding is for $\tilde{\mathcal{F}}_{x, D}, \mathcal{F}_{x, \psi}$, etc.

Lemma 2.3. Let $D \subseteq X, H \subseteq X^{*}$, and $E \subseteq X^{*} \times \mathbb{R}$ be convex subsets. Then the following formulas hold for each $x \in D$ :

$$
\bigcap_{L \in \tilde{\mathcal{F}}_{x, D}} \operatorname{cl}\left(E+\operatorname{epi} \sigma_{L \cap D}\right)=\bigcap_{L \in \mathcal{F}_{x, D}} \operatorname{cl}\left(E+\operatorname{epi} \sigma_{L \cap D}\right)=\bigcap_{L \in \mathcal{F}_{D}} \operatorname{cl}\left(E+\operatorname{epi} \sigma_{L \cap D}\right)
$$

and

$$
\bigcap_{L \in \tilde{\mathcal{F}}_{x, D}} \operatorname{cl}\left(H+\mathrm{N}_{L \cap D}(x)\right)=\bigcap_{L \in \mathcal{F}_{x, D}} \operatorname{cl}\left(H+\mathrm{N}_{L \cap D}(x)\right) .
$$

Furthermore, if additionally ri $D \neq \varnothing$, then the following formulas hold:

Copyright ( $\odot$ by SIAM. Unauthorized reproduction of this article is prohibited. 


$$
\bigcap_{L \in \mathcal{F}_{D}} \operatorname{cl}\left(E+\operatorname{epi} \sigma_{L \cap D}\right)=\operatorname{cl}\left(E+\operatorname{epi} \sigma_{D}\right)
$$

and

$$
\bigcap_{L \in \mathcal{F}_{x, D}} \operatorname{cl}\left(H+\mathrm{N}_{L \cap D}(x)\right)=\operatorname{cl}\left(H+\mathrm{N}_{D}(x)\right) \quad \text { for each } x \in D .
$$

Proof. Let $x \in D$. Evidently, $\mathcal{F}_{x, D} \subseteq \mathcal{F}_{D}$, and on the other hand, for any $L \in \mathcal{F}_{D}$ and $L_{x}:=\operatorname{span}(L \cup\{x\})$, one has $L_{x} \in \mathcal{F}_{x, D}$ and epi $\sigma_{L_{x} \cap D} \subseteq$ epi $\sigma_{L \cap D}$. Thus the second equality in (2.11) is clear. For the first equality, it suffices to verify that

$$
\bigcap_{L \in \tilde{\mathcal{F}}_{x, D}} \operatorname{cl}\left(E+\operatorname{epi} \sigma_{L \cap D}\right) \supseteq \bigcap_{L \in \mathcal{F}_{x, D}} \operatorname{cl}\left(E+\operatorname{epi} \sigma_{L \cap D}\right),
$$

because the converse inclusion holds automatically (noting $\mathcal{F}_{x, D} \subseteq \tilde{\mathcal{F}}_{x, D}$ ). To establish (2.15), let $V \subseteq X^{*}$ be a weak* neighborhood of 0 . Let $L^{\prime} \in \tilde{\mathcal{F}}_{x, D}$ and choose $L_{0} \in \mathcal{F}_{x, D}$ such that $L_{0}^{\perp} \subseteq V$. Without loss of generality, we assume that $L_{0} \cap \operatorname{ri}\left(L^{\prime} \cap D\right) \neq \varnothing$ (replaced by the span generated by $L_{0}$ with an element of $\operatorname{ri}\left(L^{\prime} \cap D\right)$ if necessary). It follows from (2.9) that

$$
\text { epi } \sigma_{L_{0} \cap\left(L^{\prime} \cap D\right)}=\operatorname{cl}\left(\operatorname{epi} \sigma_{L_{0}}+\operatorname{epi} \sigma_{L^{\prime} \cap D}\right)=\operatorname{cl}\left(L_{0}^{\perp} \times \mathbb{R}_{+}+\operatorname{epi} \sigma_{L^{\prime} \cap D}\right),
$$

where $\mathbb{R}_{+}:=[0,+\infty)$ as usual. Hence, for any $\delta>0$,

$$
\begin{aligned}
\bigcap_{L \in \mathcal{F}_{x, D}} \operatorname{cl}\left(E+\operatorname{epi} \sigma_{L \cap D}\right) & \subseteq \operatorname{cl}\left(E+\operatorname{epi} \sigma_{L_{0} \cap\left(L^{\prime} \cap D\right)}\right) \\
& =\operatorname{cl}\left(E+L_{0}^{\perp} \times \mathbb{R}_{+}+\operatorname{epi} \sigma_{L^{\prime} \cap D}\right) \\
& \subseteq E+\operatorname{epi} \sigma_{L^{\prime} \cap D}+2 V \times(-\delta,+\delta) .
\end{aligned}
$$

Since $V$ is an arbitrary neighborhood, we get that

$$
\bigcap_{L \in \mathcal{F}_{x, D}} \operatorname{cl}\left(E+\operatorname{epi} \sigma_{L^{\prime} \cap D}\right) \subseteq \bigcap_{V \in \mathcal{V}, \delta>0}\left(E+\operatorname{epi} \sigma_{L^{\prime} \cap D}+V \times(-\delta,+\delta)\right)=\operatorname{cl}\left(E+\operatorname{epi} \sigma_{L^{\prime} \cap D}\right),
$$

where $\mathcal{V}$ denotes the set of all weak* neighborhoods of 0 . Since $L^{\prime} \in \tilde{\mathcal{F}}_{x, D}$ is arbitrary, it follows that (2.15) holds.

Now assume that ri $D \neq \varnothing$. Let $L_{0}:=\operatorname{span} D$. Then $L_{0} \in \tilde{\mathcal{F}}_{x, D}$ and $L_{0} \cap D=D$. Hence

$$
\bigcap_{L \in \tilde{\mathcal{F}}_{x, D}} \operatorname{cl}\left(E+\operatorname{epi} \sigma_{L \cap D}\right) \subseteq \operatorname{cl}\left(E+\operatorname{epi} \sigma_{L_{0} \cap D}\right)=\operatorname{cl}\left(E+\operatorname{epi} \sigma_{D}\right)
$$

The converse inclusion is clear because $\operatorname{cl}\left(E+\operatorname{epi} \sigma_{D}\right) \subseteq \operatorname{cl}\left(E+\operatorname{epi} \sigma_{L \cap D}\right)$ for any $L \in \tilde{\mathcal{F}}_{x, D}$. Thus, together with (2.11), we see that (2.13) is true. Assertions regarding (2.12) and (2.14) are proved similarly, but one applies (2.10) in place of (2.9).

2.2. Supremum functions. In the remainder of this paper, let $\left\{f_{i}: i \in I\right\}$ denote a family of proper convex functions on $X$, where $I$ is an index set. Let $\psi:=\sup _{i \in I} f_{i}$ denote the sup-function of $\left\{f_{i}: i \in I\right\}$, that is,

$$
\psi(x):=\sup \left\{f_{i}(x): i \in I\right\} \quad \text { for each } x \in X .
$$

Copyright ( by SIAM. Unauthorized reproduction of this article is prohibited. 
The sup-function of $\left\{\mathrm{cl} f_{i}: i \in I\right\}$ will be denoted by $\psi_{\mathrm{cl}}$, that is,

$$
\psi_{\mathrm{cl}}(x):=\sup \left\{\left(\operatorname{cl} f_{i}\right)(x): i \in I\right\} \quad \text { for each } x \in X .
$$

Note that $\psi_{\mathrm{cl}}$ is lower semicontinuous, $\psi_{\mathrm{cl}} \leq \psi$, and so

$$
\psi_{\mathrm{cl}} \leq \mathrm{cl} \psi \leq \psi .
$$

We always assume that $\psi$ and $\psi_{\mathrm{cl}}$ are proper. Lemma 2.4 below is known in [19].

LEMMA 2.4. The following assertion regarding epigraphs holds:

$$
\operatorname{epi} \psi_{\mathrm{cl}}^{*}=\operatorname{cl}\left(\operatorname{co} \bigcup_{i \in I} \operatorname{epi} f_{i}^{*}\right) \text {. }
$$

For a convex set $D$ in $X$ and a proper convex function $F$ on $X$, we say that $(D, F)$ is an associate pair of convex set and convex function for $\left\{f_{i}: i \in I\right\}$ if

$$
\left.F\right|_{D}=\left.\psi\right|_{D} \text { and } \operatorname{dom} F \subseteq D \subseteq \operatorname{dom} \psi_{\mathrm{cl}} .
$$

Remark 2.1. (dom $\psi, \psi)$ and $\left(\operatorname{dom} \psi_{\mathrm{cl}}, \psi\right)$ are examples of such pairs.

Remark 2.2. The condition (2.18) implies that

$$
\begin{gathered}
\operatorname{dom} F=D \cap \operatorname{dom} \psi, \quad F=\psi+\delta_{D}, \\
\operatorname{epi} F^{*} \supseteq \bigcap_{L \in \mathcal{F}_{D}} \operatorname{cl}\left(\operatorname{co~} \cup_{i \in I} \operatorname{epi} f_{i}^{*}+\operatorname{epi} \sigma_{L \cap D}\right) \supseteq \operatorname{cl}\left(\operatorname{co~} \cup_{i \in I} \text { epi } f_{i}^{*}+\operatorname{epi} \sigma_{D}\right),
\end{gathered}
$$

and that

$$
(\operatorname{cl} F)(x) \geq \sup _{i \in I}\left(\operatorname{cl} f_{i}\right)(x) \quad \text { for each } x \in X .
$$

Indeed, (2.19) and the second inclusion of (2.20) are evident (assuming (2.18)). By definition and (2.19), it is easily seen that $\psi+\delta_{D} \geq f_{i}$, i.e., $F \geq f_{i}$ and so $\operatorname{cl} F \geq \operatorname{cl} f_{i}$ for each $i$; thus (2.21) is also clear. By a direct verification together with the fact that $D=$ $\cup_{L \in \mathcal{F}_{D}}(L \cap D)$ we have $\left(\psi+\delta_{D}\right)^{*}=\sup _{L \in \mathcal{F}_{D}}\left(\psi+\delta_{L \cap D}\right)^{*}$, and so it follows from (2.19) that

$$
\operatorname{epi} F^{*}=\operatorname{epi}\left(\sup _{L \in \mathcal{F}_{D}}\left(\psi+\delta_{L \cap D}\right)^{*}\right)=\bigcap_{L \in \mathcal{F}_{D}} \operatorname{epi}\left(\psi+\delta_{L \cap D}\right)^{*}
$$

This implies that the first inclusion of (2.20) holds since, for each $L \in \mathcal{F}_{D}$, $\operatorname{epi}\left(\psi+\delta_{L \cap D}\right)^{*}$ is weak* closed, convex, and

$$
\operatorname{epi}\left(\psi+\delta_{L \cap D}\right)^{*} \supseteq \operatorname{epi} \psi^{*}+\operatorname{epi} \delta_{L \cap D}^{*} \supseteq \operatorname{epi} f_{i}^{*}+\operatorname{epi} \sigma_{L \cap D} \quad \text { for each } i \in I .
$$

The next proposition addresses the following question: When do the inclusions/ inequalities in (2.20) and (2.21) become equalities?

Proposition 2.5. Let $(D, F)$ be an associate pair of convex set and convex function for $\left\{f_{i}: i \in I\right\}$. Consider the following statements:

$$
\text { epi } F^{*}=\operatorname{cl}\left(\operatorname{co} \cup_{i \in I} \text { epi } f_{i}^{*}+\operatorname{epi} \sigma_{D}\right) .
$$

Copyright ( by SIAM. Unauthorized reproduction of this article is prohibited. 


$$
\begin{gathered}
(\operatorname{cl} F)(x)=\sup _{i \in I}\left(\operatorname{cl} f_{i}\right)(x) \quad \text { for each } x \in \operatorname{cl} D . \\
\operatorname{epi} F^{*}=\bigcap_{L \in \mathcal{F}_{D}} \operatorname{cl}\left(\operatorname{co} \cup_{i \in I} \text { epi } f_{i}^{*}+\operatorname{epi} \sigma_{L \cap D}\right) . \\
(\operatorname{cl} F)(x)=\sup _{i \in I}\left(\operatorname{cl} f_{i}\right)(x) \quad \text { for each } x \in \bigcup_{L \in \mathcal{F}_{D}} \operatorname{cl}(L \cap D) .
\end{gathered}
$$

Then the following implications/equivalences hold:

$$
(2.22) \Leftrightarrow(2.23) \Rightarrow(2.24) \Leftrightarrow(2.25) .
$$

Proof. In view of (2.20) and (2.21), we have the following chain of equivalences:

$$
\begin{aligned}
(2.25) & \Leftrightarrow(\operatorname{cl} F)(x) \leq \sup _{i \in I}\left(\operatorname{cl} f_{i}\right)(x) \quad \text { for each } x \in \operatorname{cl}(L \cap D) \quad \text { and } \quad L \in \mathcal{F}_{D} \\
& \Leftrightarrow(\operatorname{cl} F) \leq \sup _{i \in I}\left(\operatorname{cl} f_{i}+\delta_{\operatorname{cl}(L \cap D)}\right) \quad \text { for each } L \in \mathcal{F}_{D} \\
& \Leftrightarrow \operatorname{epi} F^{*} \subseteq \operatorname{cl}\left(\operatorname{co~} \cup_{i \in I} \operatorname{epi}\left(\operatorname{cl} f_{i}+\delta_{\operatorname{cl}(L \cap D)}\right)^{*}\right) \quad \text { for each } L \in \mathcal{F}_{D} \\
& \Leftrightarrow \operatorname{epi} F^{*} \subseteq \operatorname{cl}\left(\operatorname{co~} \cup_{i \in I} \operatorname{epi} f_{i}^{*}+\operatorname{epi} \sigma_{L \cap D}\right) \quad \text { for each } L \in \mathcal{F}_{D} \\
& \Leftrightarrow(2.24),
\end{aligned}
$$

where the third equivalence holds because of (2.2) and Lemma (2.4), and the fourth equivalence holds because, by Lemma 2.1,

$$
\operatorname{epi}\left(\operatorname{cl} f_{i}+\delta_{\mathrm{cl}(L \cap D)}\right)^{*}=\operatorname{cl}\left(\operatorname{epi}\left(\operatorname{cl} f_{i}\right)^{*}+\operatorname{epi} \sigma_{\mathrm{cl}(L \cap D)}\right)=\operatorname{cl}\left(\operatorname{epi} f_{i}^{*}+\operatorname{epi} \sigma_{L \cap D}\right) .
$$

A similar argument shows $(2.22) \Leftrightarrow(2.23)$. Since implication $(2.23) \Rightarrow(2.25)$ is trivial, the proof is complete.

Remark 2.3. In view of Lemma 2.3 (applied to co $\cup_{i \in I}$ epi $f_{i}^{*}$ in place of $E$ ), we see that if ri $D \neq \varnothing$, then statements (2.22)-(2.25) are equivalent.

Corollary 2.6. The following equalities are equivalent:

$$
\begin{gathered}
\text { epi } \psi^{*}=\bigcap_{L \in \mathcal{F}_{\mathrm{cl} \psi}} \operatorname{cl}\left(\operatorname{co} \cup_{i \in I} \operatorname{epi} f_{i}^{*}+\operatorname{epi} \sigma_{L \cap \operatorname{dom} \psi_{\mathrm{cl}}}\right) \text {. } \\
\text { epi } \psi^{*}=\operatorname{cl}\left(\operatorname{co} \cup_{i \in I} \operatorname{epi} f_{i}^{*}+\operatorname{epi} \sigma_{\operatorname{dom} \psi_{\mathrm{cl}}}\right) \text {. } \\
\operatorname{epi} \psi^{*}=\operatorname{cl}\left(\operatorname{co} \cup_{i \in I} \operatorname{epi} f_{i}^{*}\right) . \\
(\operatorname{cl} \psi)(x)=\sup _{i \in I}\left(\operatorname{cl} f_{i}\right)(x) \quad \text { for each } x \in X . \\
(\operatorname{cl} \psi)(x)=\sup _{i \in I}\left(\operatorname{cl} f_{i}\right)(x) \quad \text { for each } x \in \operatorname{dom} \psi_{\mathrm{cl}} .
\end{gathered}
$$

Proof. By Proposition 2.5 (applied to $\left(\operatorname{dom} \psi_{\mathrm{cl}}, \psi\right)$ in place of $\left.(D, F)\right),(2.26)$ implies (in fact, is equivalent to) that

$$
(\operatorname{cl} \psi)(x)=\sup _{i \in I}\left(\operatorname{cl} f_{i}\right)(x) \quad \text { for each } x \in \bigcup_{L \in \mathcal{F}_{\mathrm{cl} \psi}} \operatorname{cl}\left(L \cap \operatorname{dom} \psi_{\mathrm{cl}}\right),
$$

which in turn implies (2.30) because $\operatorname{dom} \psi_{\mathrm{cl}} \subseteq \bigcup_{L \in \mathcal{F}_{\mathrm{cl} \psi}} \operatorname{cl}\left(L \cap \operatorname{dom} \psi_{\mathrm{cl}}\right)$. The equivalence $(2.29) \Leftrightarrow(2.30)$ is evident because, by $(2.16),+\infty=\psi_{\mathrm{cl}} \leq \mathrm{cl} \psi$ on $X \backslash \operatorname{dom} \psi_{\mathrm{cl}}$. Since (2.29) means that $\mathrm{cl} \psi=\psi_{\mathrm{cl}}$ (which in turn entails that epi $\psi^{*}=\operatorname{epi} \psi_{\mathrm{cl}}^{*}$, the

Copyright ( by SIAM. Unauthorized reproduction of this article is prohibited. 
set expressed on the right-hand side of (2.28) by Lemma 2.4), it follows that $(2.29) \Rightarrow(2.28)$. Hence the proof is complete as implications $(2.28) \Rightarrow(2.27) \Rightarrow$ (2.26) are trivially true after taking into account (2.20).

3. Subdifferentials of supremum functions. Let $x \in X$ and $\epsilon>0$. We define

$$
I_{\epsilon}(x):=\left\{i \in I: f_{i}(x) \geq \psi(x)-\epsilon\right\}
$$

and

$$
I(x):=I_{0}(x)=\left\{i \in I: f_{i}(x)=\psi(x)\right\} .
$$

We are now ready to present our main result below. In view of Remark 2.1, this result is applicable for the special pairs $(\operatorname{dom} \psi, \psi)$ and $\left(\operatorname{dom} \psi_{\mathrm{cl}}, \psi\right)$, and thereby we establish some equivalent conditions ensuring the subdifferential calculus rules for supremum functions to hold.

Theorem 3.1. Let $(D, F)$ be an associate pair of convex set and convex function for $\left\{f_{i}: i \in I\right\}$. Let $\eta>0$. Then the following formulas are equivalent for any $x \in D$ :

$$
\begin{gathered}
(\partial F(x) \times \mathbb{R}) \cap \operatorname{epi} F^{*} \subseteq \bigcap_{L \in \mathcal{F}_{D}} \operatorname{cl}\left(\operatorname{co~} \cup_{i \in I} \text { epi } f_{i}^{*}+\operatorname{epi} \sigma_{L \cap D}\right) . \\
\partial F(x)=\bigcap_{L \in \mathcal{F}_{x, D}, \epsilon>0} \operatorname{cl}\left(\operatorname{co~} \cup_{i \in I_{\epsilon}(x)} \partial_{\eta \epsilon} f_{i}(x)+\mathrm{N}_{L \cap D}(x)\right) .
\end{gathered}
$$

Moreover, if additionally $X$ is assumed to be a Banach space, then formula (3.2) holds for each $x \in D$ if and only if the following statement holds:

$$
(\operatorname{cl} F)(x)=\sup _{i \in I}\left(\operatorname{cl} f_{i}\right)(x) \quad \text { for each } x \in \bigcup_{L \in \mathcal{F}_{D}} \operatorname{cl}(L \cap D) .
$$

Proof. For the proof of (3.1) $\Leftrightarrow(3.2)$, let $x \in D$. We may assume that $x \in \operatorname{dom} F$ (otherwise, $x \notin \operatorname{dom} \psi$ by (2.18) and $x \notin \operatorname{dom} f_{i}$ for each $i \in I_{\epsilon}(x)$ with $\epsilon>0$, and consequently, formulas (3.1) and (3.2) hold trivially since $\partial F(x)$ and each $\partial_{\eta_{\epsilon}} f_{i}(x)$ are empty).

$[(3.1) \Rightarrow(3.2)]$. For any $\epsilon>0$ and $L \in \mathcal{F}_{x, D}$, set

$$
H_{L, \epsilon}:=\operatorname{cl}\left(\operatorname{co} \cup_{i \in I_{\epsilon}(x)} \partial_{\eta \epsilon} f_{i}(x)+\mathrm{N}_{L \cap D}(x)\right) .
$$

Clearly, (3.2) can be rewritten as

$$
\partial F(x)=\bigcap_{L \in \mathcal{F}_{x, D}, \epsilon>0} H_{L, \epsilon} .
$$

Noting (2.18) and that $\operatorname{dom} F \subseteq D=\cup_{L \in \mathcal{F}_{x, D}} L \cap D$, it is not difficult to verify that

$$
\partial_{(1+\eta) \epsilon} F(x) \supseteq \bigcap_{L \in \mathcal{F}_{x, D}} H_{L, \epsilon} .
$$

Since $\partial F(x)=\bigcap_{\alpha>0} \partial_{\alpha} F(x)$, we see that, to show (3.4), it is sufficient to show that

$$
\partial F(x) \subseteq \bigcap_{L \in \mathcal{F}_{x, D}, \epsilon>0} H_{L, \epsilon} .
$$

To do this, let $x^{*} \in \partial F(x), L \in \mathcal{F}_{x, D}$, and $\epsilon>0$. For (3.5), it suffices to show that

$$
x^{*} \in H_{L, \epsilon} .
$$

Copyright ( by SIAM. Unauthorized reproduction of this article is prohibited. 
Set

$$
\alpha:=\left\langle x^{*}, x\right\rangle-F(x)=\left\langle x^{*}, x\right\rangle-\psi(x) .
$$

Then, by $(2.6),\left(x^{*}, \alpha\right) \in(\partial F(x) \times \mathbb{R}) \cap$ epi $F^{*}$. By $(3.1)$,

$$
\left(x^{*}, \alpha\right) \in \operatorname{cl}\left(\operatorname{co} \cup_{i \in I} \operatorname{epi} f_{i}^{*}+\operatorname{epi} \sigma_{L \cap D}\right),
$$

so that there exists a net $\left\{\left(x_{\tau}^{*}, \alpha_{\tau}\right)\right\} \subseteq \operatorname{co\bigcup } \bigcup_{i \in I}$ epi $f_{i}^{*}+\operatorname{epi} \sigma_{L \cap D}$ such that

$$
x_{\tau}^{*} \rightarrow x^{*} \quad \text { and } \quad \alpha_{\tau} \rightarrow \alpha,
$$

and each $\left(x_{\tau}^{*}, \alpha_{\tau}\right)$ can be represented in the form

$$
\left(x_{\tau}^{*}, \alpha_{\tau}\right)=\sum_{j \in J_{\tau}} t_{\tau}^{j}\left(x^{*}{ }_{\tau}^{j}, \alpha_{\tau}^{j}\right)+\left(y_{\tau}^{*}, \alpha_{\tau}^{0}\right),
$$

where $\varnothing \neq J_{\tau} \subseteq I$ is finite, $\left\{f_{\tau}^{j}: j \in J_{\tau}\right\} \subseteq\left\{f_{i}: i \in I\right\}$,

$$
\left(y_{\tau}^{*}, \alpha_{\tau}^{0}\right) \in \operatorname{epi} \sigma_{L \cap D}, \quad\left(x_{\tau}^{* j}, \alpha_{\tau}^{j}\right) \in \operatorname{epi} f_{\tau}^{j *}, \quad \text { and } \quad t_{\tau}^{j}>0 \quad \text { for each } j \in J_{\tau}
$$

with $\sum_{j \in J_{\tau}} t_{\tau}^{j}=1$. Write

$$
\hat{L}_{0}:=\operatorname{cl}(\operatorname{cone}(L \cap D-x)) .
$$

Then $\hat{L}_{0}^{\ominus}=\mathrm{N}_{L \cap D}(x)$ and

$$
z_{0}^{*}+\hat{L}_{0}^{\ominus} \subseteq H_{L, \epsilon} \quad \text { for each } z_{0}^{*} \in H_{L, \epsilon} .
$$

We will construct a net $\left\{w_{\tau}^{*}\right\} \subseteq H_{L, \epsilon}$ satisfying the property that

$$
\left\langle x^{*}, y\right\rangle \leq \lim \sup _{\tau}\left\langle w_{\tau}^{*}, y\right\rangle \quad \text { for each } y \in \hat{L}_{0} .
$$

If this is the case, then (3.6) holds. Indeed, if $x^{*} \notin H_{L, \epsilon}$, it would follow from the separation theorem that, for some $\bar{y} \in X$,

$$
\left\langle x^{*}, \bar{y}\right\rangle>\sup _{w^{*} \in H_{L, e}}\left\langle w^{*}, \bar{y}\right\rangle .
$$

Pick $z_{0}^{*} \in H_{L, \epsilon}$ and it follows from (3.11) that

$$
\left\langle x^{*}, \bar{y}\right\rangle>\left\langle z_{0}^{*}, \bar{y}\right\rangle+\sup _{z^{*} \in \hat{L}_{0}^{\ominus}}\left\langle z^{*}, \bar{y}\right\rangle,
$$

which entails that $\bar{y} \in \hat{L}_{0}$ because $\hat{L}_{0}$ is a closed convex cone. But (3.13) and (3.12) (with $y=\bar{y}$ ) are not consistent as $\left\{w_{\tau}^{*}\right\} \subseteq H_{L, \epsilon}$.

Therefore we need only to find a net $\left\{w_{\tau}^{*}\right\} \subseteq H_{L, \epsilon}$ with the stated property. To do this, we first pick $a \in \operatorname{ri}(L \cap D)$ and, for simplicity, write $L_{0}:=\operatorname{span}(L \cap D-x)$. Clearly,

$$
\hat{L}_{0} \subseteq L_{0}=\operatorname{cone}(L \cap D-a) .
$$

Without loss of generality, we assume that the convergent nets $\left\{\alpha_{\tau}\right\}$ and $\left\{\left\langle x_{\tau}^{*}, a\right\rangle\right\}$ are bounded. Since $D \subseteq \operatorname{dom} \psi_{\mathrm{cl}}$ by (2.18), we regard each $\alpha_{\tau}-x_{\tau}^{*}-\psi_{\mathrm{cl}}$ as a function on $L \cap D$, and we can use (3.9) to represent this function as follows:

Copyright $@$ by SIAM. Unauthorized reproduction of this article is prohibited. 


$$
\alpha_{\tau}-x_{\tau}^{*}+\psi_{\mathrm{cl}}=\left(\alpha_{\tau}^{0}-y_{\tau}^{*}\right)+\sum_{j \in J_{\tau}} t_{\tau}^{j}\left(\alpha_{\tau}^{j}-x^{*}{ }_{\tau}^{j}+\operatorname{cl} f_{\tau}^{j}\right)+\sum_{j \in J_{\tau}} t_{\tau}^{j}\left(\psi_{\mathrm{cl}}-\operatorname{cl} f_{\tau}^{j}\right) .
$$

By (3.10) and the definition of $\psi_{\mathrm{cl}}$, each term of the above summations is nonnegative, i.e.,

$$
\left(\alpha_{\tau}^{0}-y_{\tau}^{*}\right) \geq 0, \quad\left(\alpha_{\tau}^{j}-x_{\tau}^{*}+\operatorname{cl} f_{\tau}^{j}\right) \geq 0, \quad \text { and } \quad\left(\psi_{\mathrm{cl}}-\operatorname{cl} f_{\tau}^{j}\right) \geq 0 \quad \text { on } L \cap D .
$$

Since $a \in L \cap D$, it follows from (3.15) that

$$
0 \leq \alpha_{\tau}^{0}-\left\langle y_{\tau}^{*}, a\right\rangle \leq \alpha_{\tau}-\left\langle x_{\tau}^{*}, a\right\rangle+\psi_{\mathrm{cl}}(a),
$$

and so the net $\left\{\alpha_{\tau}^{0}-\left\langle y_{\tau}^{*}, a\right\rangle\right\}$ is bounded as $\left\{\alpha_{\tau}\right\}$ and $\left\{\left\langle x_{\tau}^{*}, a\right\rangle\right\}$ are. Consequently, $\left\{\left\langle y_{\tau}^{*}, y\right\rangle\right\}$ is bounded above for any $y \in L \cap D-a$ because, for any $z \in L \cap D$, one has by $(3.16)$

$$
\left\langle y_{\tau}^{*}, z-a\right\rangle \leq \alpha_{\tau}^{0}-\left\langle y_{\tau}^{*}, a\right\rangle \text { for all } \tau .
$$

Therefore, the net $\left\{y_{\tau}^{*}\right\}$ of linear functionals is bounded above on $L_{0}$ (see (3.14)). Thus, without loss of generality, we may assume that there exists $y_{0}^{*} \in X^{*}$ such that

$$
\lim _{\tau}\left\langle y_{\tau}^{*}, v\right\rangle=\left\langle y_{0}^{*}, v\right\rangle \quad \text { for each } v \in L_{0} .
$$

On the other hand, note that $f_{j}(x) \leq \psi(x)=F(x)<+\infty$ (since $x \in \operatorname{dom} F$ as assumed at the beginning of the proof). Similar to (3.15) and (3.16), we have

$$
\begin{aligned}
& \left(\alpha_{\tau}-x_{\tau}^{*}+\psi\right)(x)=\left(\alpha_{\tau}^{0}-\left\langle y_{\tau}^{*}, x\right\rangle\right)+\sum_{j \in J_{\tau}} t_{\tau}^{j}\left(\alpha_{\tau}^{j}-\left\langle x_{\tau}^{*}{ }_{\tau}^{j}, x\right\rangle+f_{\tau}^{j}(x)\right)+\sum_{j \in J_{\tau}} t_{\tau}^{j}\left(\psi(x)-f_{\tau}^{j}(x)\right), \\
& 0 \leq\left(\alpha_{\tau}^{0}-\left\langle y_{\tau}^{*}, x\right\rangle\right) \\
& 0 \leq t_{\tau}^{j}\left(\alpha_{\tau}^{j}-\left\langle x_{\tau}^{*}, x\right\rangle+f_{\tau}^{j}(x)\right) \text {, }
\end{aligned}
$$

and

$$
0 \leq t_{\tau}^{j}\left(\psi(x)-f_{\tau}^{j}(x)\right)
$$

Recalling from (3.8) and our choice of $\alpha$, we also have

$$
\lim _{\tau}\left(\alpha_{\tau}-x_{\tau}^{*}+\psi\right)(x)=\left(\alpha-x^{*}+\psi\right)(x)=0,
$$

and it follows that the quantity on the right-hand side of each (3.20)-(3.22) converges to zero and for any constant $\xi>0$,

$$
\lim _{\tau}\left(\sum_{j \in J_{\tau}(\xi)} t_{\tau}^{j}\right)=0
$$

where

$$
J_{\tau}(\xi):=\left\{j \in J_{\tau}: \xi \leq \alpha_{\tau}^{j}-\left\langle x_{\tau}^{* j}, x\right\rangle+f_{\tau}^{j}(x) \quad \text { or } \quad \xi \leq \psi(x)-f_{\tau}^{j}(x)\right\} .
$$

Copyright ( by SIAM. Unauthorized reproduction of this article is prohibited. 
Let us fix $\xi:=\min \{\epsilon, \eta \epsilon\}$. Denote $\lambda_{\tau}:=\sum\left\{t_{\tau}^{j}: j \in J_{\tau} \backslash J_{\tau}(\xi)\right\}$ and $z_{\tau}^{*}:=\frac{1}{\lambda_{\tau}} \sum\left\{t_{\tau}^{j} x_{\tau}^{* j}: j \in\right.$ $\left.J_{\tau} \backslash J_{\tau}(\xi)\right\}$. Since $\sum_{j \in J_{\tau}} t_{\tau}^{j}=1$, it follows from (3.24) that $\lambda_{\tau} \rightarrow 1$, and so we may assume (as we have done) that $\lambda_{\tau} \neq 0$. If $j \in J_{\tau} \backslash J_{\tau}(\xi)$, then $j \in I_{\epsilon}(x)$ and

$$
\eta \epsilon \geq \xi \geq \alpha_{\tau}^{j}-\left\langle x_{\tau}^{* j}, x\right\rangle+f_{\tau}^{j}(x) \geq\left\langle x_{\tau}^{* j}, z-x\right\rangle-f_{\tau}^{j}(z)+f_{\tau}^{j}(x)
$$

for each $z \in X$, i.e., $x^{*}{ }_{\tau}^{j} \in \partial_{\eta \epsilon} f_{\tau}^{j}(x)$. This implies that

$$
z_{\tau}^{*} \in \operatorname{co~} \cup_{i \in I_{\epsilon}(x)} \partial_{\eta \epsilon} f_{i}(x) .
$$

Note also that, since $\lim _{\tau}\left(\alpha_{\tau}^{0}-\left\langle y_{\tau}^{*}, x\right\rangle\right)=0$ as noted earlier, one has, by (3.16) and (3.18),

$$
\left\langle y_{0}^{*}, y-x\right\rangle=\lim _{\tau}\left\langle y_{\tau}^{*}, y-x\right\rangle \leq \lim _{\tau}\left(\alpha_{\tau}^{0}-\left\langle y_{\tau}^{*}, x\right\rangle\right)=0 \quad \text { for each } y \in L \cap D,
$$

that is, $y_{0}^{*} \in \mathrm{N}_{L \cap D}(x)$, and we have

$$
w_{\tau}^{*}:=z_{\tau}^{*}+y_{0}^{*} \in \operatorname{co~} \cup_{i \in I_{\epsilon}(x)} \partial_{\eta \epsilon} f_{i}(x)+\mathrm{N}_{L \cap D}(x) \subseteq H_{L, \epsilon} .
$$

Finally, we verify that the net $\left\{w_{\tau}^{*}\right\}$ satisfies (3.12). Indeed, let $z \in L \cap D$ (so $\psi_{\text {cl }}(z)<$ $+\infty$ by (2.18)). Then, for the following summation over $j \in J_{\tau}(\xi)$, one has, by (3.16),

$$
\begin{aligned}
\left\langle\sum_{j} t_{\tau}^{j} x_{\tau}^{* j}, z-x\right\rangle & \leq \sum_{j} t_{\tau}^{j}\left(\alpha_{\tau}^{j}-\left\langle x_{\tau}^{* j}, x\right\rangle+\left(\operatorname{cl} f_{\tau}^{j}\right)(z)\right) \\
& \leq \sum_{j} t_{\tau}^{j}\left(\alpha_{\tau}^{j}-\left\langle x_{\tau}^{* j}, x\right\rangle+\psi(x)\right)+\sum_{j} t_{\tau}^{j}\left(\psi_{\mathrm{cl}}(z)-\psi(x)\right),
\end{aligned}
$$

where, on the right-hand side, the first summation converges to zero by (3.23) and the second summation also does because of $(3.24)$ and $\left(\psi_{\mathrm{cl}}(z)-\psi(x)\right)$ is a finite constant. This implies that

$$
\lim \sup _{\tau} \sum_{j \in J_{\tau}(\xi)} t_{\tau}^{j}\left\langle x_{\tau}^{* j}, z-x\right\rangle \leq 0 \quad \text { for each } z \in L \cap D
$$

Since $x_{\tau}^{*}=\lambda_{\tau} z_{\tau}^{*}+y_{\tau}^{*}+\sum_{j \in J_{\tau}(\xi)} t_{\tau}^{j} x_{\tau}^{* j}$, one has

$$
\lim \sup _{\tau}\left\langle\left(x_{\tau}^{*}-\left(\lambda_{\tau} z_{\tau}^{*}+y_{\tau}^{*}\right)\right), y\right\rangle \leq 0 \text { for each } y \in \hat{L}_{0} .
$$

Since $\lambda_{\tau} \rightarrow 1$ and $\left\langle y_{\tau}^{*}, z-x\right\rangle \rightarrow\left\langle y_{0}^{*}, z-x\right\rangle$ (by (3.18) and (3.14)), it follows that

$$
\lim \sup _{\tau}\left\langle\left(x^{*}-z_{\tau}^{*}-y_{0}^{*}\right), z-x\right\rangle \leq 0 \quad \text { for all } z \in L \cap D
$$

and (3.12) follows.

$[(3.2) \Rightarrow(3.1)]$. Let $L \in \mathcal{F}_{x, D}, \epsilon>0$, and let $\left(x^{*}, \beta\right) \in(\partial F(x) \times \mathbb{R}) \cap$ epi $F^{*}$. Then $x^{*} \in \partial F(x)$ and

$$
\alpha:=\left\langle x^{*}, x\right\rangle-F(x) \leq \beta .
$$

By assumption, $x^{*} \in H_{L, \epsilon}$. Then there exists a net $\left\{x_{\tau}^{*}\right\} \subseteq \operatorname{co~} \cup_{i \in I_{\epsilon}(x)} \partial_{\eta \epsilon} f_{i}(x)+\mathrm{N}_{L \cap D}(x)$ such that

Copyright ( $\odot$ by SIAM. Unauthorized reproduction of this article is prohibited. 


$$
x_{\tau}^{*}:=\sum_{j \in J_{\tau}} t_{\tau}^{j} x_{\tau}^{* j}+y_{\tau}^{*} \stackrel{w^{*}}{\rightarrow} x^{*},
$$

where $\varnothing \neq J_{\tau} \subseteq I_{\epsilon}(x)$ is a finite subset, $\left\{f_{\tau}^{j}: j \in J_{\tau}\right\} \subseteq\left\{f_{i}: i \in I\right\}$,

$$
y_{\tau}^{*} \in \mathrm{N}_{L \cap D}(x), \quad x_{\tau}^{* j} \in \partial_{(1+\eta) \epsilon} f_{\tau}^{j}(x), \quad \text { and } \quad t_{\tau}^{j}>0 \quad \text { for each } j \in J_{\tau},
$$

satisfying that $\sum_{j \in J_{\tau}} t_{\tau}^{j}=1$ and

$$
f_{\tau}^{j}(x)>\psi(x)-\epsilon=F(x)-\epsilon \text { for each } j \in J_{\tau} .
$$

Write

$$
\alpha_{\tau}^{0}:=\left\langle y_{\tau}^{*}, x\right\rangle \quad \text { and } \quad \alpha_{\tau}^{j}:=\left\langle x_{\tau}^{*}, x\right\rangle-F(x)+(2+\eta) \epsilon \quad \text { for each } j \in J_{\tau} .
$$

Then

$$
\alpha_{\tau}^{j} \geq\left\langle x_{\tau}^{* j}, x\right\rangle-f_{\tau}^{j}(x)+(1+\eta) \epsilon,
$$

and it follows from (3.29) that

$$
\left(y_{\tau}^{*}, \alpha_{\tau}^{0}\right) \in \operatorname{epi} \sigma_{L \cap D} \quad \text { and } \quad\left(x_{\tau}^{* j}, \alpha_{\tau}^{j}\right) \in \operatorname{epi} f_{\tau}^{j *} \quad \text { for each } j \in J_{\tau} .
$$

Set

$$
\begin{aligned}
\alpha_{\tau} & :=\sum_{j \in J_{\tau}} t_{\tau}^{j} \alpha_{\tau}^{j}+\alpha_{\tau}^{0}=\left\langle\sum_{j \in J_{\tau}} t_{\tau}^{j} x_{\tau}^{* j}+y_{\tau}^{*}, x\right\rangle-F(x)+(2+\eta) \epsilon \\
& =\left\langle x_{\tau}^{*}, x\right\rangle-F(x)+(2+\eta) \epsilon
\end{aligned}
$$

and note that

$$
\alpha_{\tau} \rightarrow\left\langle x^{*}, x\right\rangle-F(x)+(2+\eta) \epsilon=\alpha+(2+\eta) \epsilon
$$

(by (3.28) and our choice of $\alpha$ ). Moreover, by (3.30) and (3.31), we have that

$$
\left(x_{\tau}^{*}, \alpha_{\tau}\right)=\sum_{j \in J_{\tau}} t_{\tau}^{j}\left(x_{\tau}^{* j}, \alpha_{\tau}^{j}\right)+\left(y_{\tau}^{*}, \alpha_{\tau}^{0}\right) \in \operatorname{co} \cup_{i \in I} \text { epi } f_{i}^{*}+\operatorname{epi} \sigma_{L \cap D} .
$$

Passing to the limits, we get

$$
\left(x^{*}, \alpha+(2+\eta) \epsilon\right) \in \operatorname{cl}\left(\operatorname{co} \cup_{i \in I} \text { epi } f_{i}^{*}+\operatorname{epi} \sigma_{L \cap D}\right) .
$$

Since $\epsilon>0$ is arbitrary and since $\alpha \leq \beta$, it follows from (3.32) that $\left(x^{*}, \beta\right) \in$ $\operatorname{cl}\left(\operatorname{co} \cup_{i \in I}\right.$ epi $\left.f_{i}^{*}+\operatorname{epi} \sigma_{L \cap D}\right)$. Since $L \in \mathcal{F}_{x, D}$ is arbitrary, the proof of the implication is complete.

For the remainder of the proof, we consider the case when $X$ is a Banach space. Note that (2.25) and (3.3) are identical, and so (3.3) is equivalent to (2.24) by Proposition 2.5. Thus we have to show that (3.2) holds for each $x \in D$ if and only if (2.24) is valid. The sufficiency part of the above required assertion follows immediately from the equivalence of (3.1) and (3.2) established above, as (2.24) evidently implies (3.1) for each $x \in D$.

Copyright ( by SIAM. Unauthorized reproduction of this article is prohibited. 
Conversely, suppose that (3.2) holds for each $x \in D$ and let $\left(x^{*}, \alpha\right) \in$ epi $F^{*}$. To show (2.24), we have to show that

$$
\left(x^{*}, \alpha\right) \in \bigcap_{L \in \mathcal{F}_{D}} \operatorname{cl}\left(\operatorname{co} \cup_{i \in I} \operatorname{epi} f_{i}^{*}+\operatorname{epi} \sigma_{L \cap D}\right) .
$$

For this purpose, we assume without loss of generality that

$$
\alpha=\sup _{x \in X}\left(\left\langle x^{*}, x\right\rangle-F(x)\right)=F^{*}\left(x^{*}\right) .
$$

Since $X$ is a Banach space, one can apply [29, Theorem 3.1.4(ii)] and there exist sequences $\left\{x_{n}\right\} \subseteq X$ and $\left\{x_{n}^{*}\right\} \subseteq X^{*}$ with each $x_{n}^{*} \in \partial F\left(x_{n}\right)$ such that

$$
\left\|x_{n}^{*}-x^{*}\right\| \rightarrow 0 \quad \text { and } \quad F^{*}\left(x_{n}^{*}\right) \rightarrow F^{*}\left(x^{*}\right) .
$$

Let $n \in \mathbb{N}$. By $(2.5)$, we have that

$$
\alpha_{n}:=\left\langle x_{n}^{*}, x_{n}\right\rangle-F\left(x_{n}\right)=F^{*}\left(x_{n}^{*}\right) .
$$

Combining (3.34), (3.35), and (3.36), we obtain that $\left(x_{n}^{*}, \alpha_{n}\right) \rightarrow\left(x^{*}, \alpha\right)$. Clearly, $\left(x_{n}^{*}, \alpha_{n}\right) \in\left(\partial F\left(x_{n}\right) \times \mathbb{R}\right) \cap$ epi $F^{*}$. Since the formula (3.2) holds at $x_{n}$ in place of $x$, it follows from the equivalence of (3.1) and (3.2) established above that

$$
\left(x_{n}^{*}, \alpha_{n}\right) \in \bigcap_{L \in \mathcal{F}_{D}} \operatorname{cl}\left(\operatorname{co} \cup_{i \in I} \operatorname{epi} f_{i}^{*}+\operatorname{epi} \sigma_{L \cap D}\right) .
$$

Thus (3.33) follows as required to show.

Remark 3.1. Suppose that ri $D \neq \varnothing$. Then by Lemma 2.3 (applied to co $\cup_{i \in I_{\epsilon}(x)} \partial_{\eta \epsilon} f_{i}(x)$ in place of $H$ ) and Remark 2.3, a variant version of Theorem 3.1 also holds in which $\cap_{L \in \mathcal{F}_{D}} \operatorname{cl}\left(\operatorname{co} \cup_{i \in I}\right.$ epi $\left.f_{i}^{*}+\operatorname{epi} \sigma_{L \cap D}\right)$ in (3.1) is replaced by $\operatorname{cl}\left(\operatorname{co} \cup_{i \in I}\right.$ epi $\left.f_{i}^{*}+\operatorname{epi} \sigma_{D}\right), \cap_{L \in \mathcal{F}_{x . D}, \epsilon>0} \operatorname{cl}\left(\operatorname{co~} \cup_{i \in I_{\epsilon}(x)} \partial_{\eta \epsilon} f_{i}(x)+\mathrm{N}_{L \cap D}(x)\right)$ in (3.2) is replaced by $\cap_{\epsilon>0} \operatorname{cl}\left(\operatorname{co} \cup_{i \in I_{\epsilon}(x)} \partial_{\eta \epsilon} f_{i}(x)+\mathrm{N}_{D}(x)\right)$, and $\cup_{L \in \mathcal{F}_{D}} \operatorname{cl}(L \cap D)$ in (3.3) is replaced by cl $D$.

Part (ii) of the following corollary was inaccurately stated (see a counterexample given at the end of this section) in [11, Corollary 8] without assumption (3.37), which was used indeed in its proof.

Corollary 3.2. Suppose that

$$
(\operatorname{cl} \psi)(x)=\sup _{i \in I}\left(\operatorname{cl} f_{i}\right)(x) \quad \text { for each } x \in \operatorname{cl}(\operatorname{dom} \psi) .
$$

Let $\eta>0$. Then the following assertions hold:

(i) The formula

$$
\partial \psi(x)=\bigcap_{L \in \mathcal{F}_{x, \psi}, \epsilon>0} \operatorname{cl}\left(\operatorname{co} \cup_{i \in I_{\epsilon}(x)} \partial_{\eta \epsilon} f_{i}(x)+\mathrm{N}_{L \cap \operatorname{dom} \psi}(x)\right)
$$

holds for each $x \in \operatorname{dom} \psi$.

(ii) If additionally $\operatorname{ri}(\operatorname{dom} \psi) \neq \varnothing$, then the formula

$$
\partial \psi(x)=\bigcap_{\epsilon>0} \operatorname{cl}\left(\operatorname{co} \cup_{i \in I_{\epsilon}(x)} \partial_{\eta \epsilon} f_{i}(x)+\mathrm{N}_{\mathrm{dom} \psi}(x)\right)
$$

holds for each $x \in \operatorname{dom} \psi$.

Proof. Take $F=\psi$ and $D=\operatorname{dom} \psi$. Thanks to assumption (3.37), (2.23) holds (and so does (2.22) by Proposition 2.5). Thus, a fortiori, (3.1) holds for each $x \in D$

Copyright ( by SIAM. Unauthorized reproduction of this article is prohibited. 
and so does (3.2) by Theorem 3.1. This means that (i) is true. Moreover, if $\operatorname{ri}(\operatorname{dom} \psi) \neq \varnothing$, then the set expressed on the right-hand side of (3.38) and that of (3.39) are equal (see Lemma 2.3) and so (ii) holds by (i).

We end this section with two simple examples. The first example shows that the assumption (3.37) in Corollary 3.2 cannot be dropped, and the second example demonstrates a case that our result is applicable but not the main result in [11], i.e., [11, Theorem 4].

Example 3.1. Let $X=\mathbb{R}$ and $I=\mathbb{N}$. Consider the family $\left\{f_{i}: i \in I\right\}$ defined by

$$
f_{i}(x):=\left\{\begin{array}{cc}
(-i) x-1, & x \in\left(-\infty,-\frac{1}{i}\right], \\
0, & x \in\left[-\frac{1}{i}, 0\right), \\
1, & x=0, \\
+\infty, & x \in(0,+\infty),
\end{array} \text { for each } i \in I .\right.
$$

Then $\psi=\delta_{\{0\}}+1$, and so $\partial \psi(0)=\mathbb{R}$. Moreover, for any $0 \leq \epsilon<1$ and $i \in I$, $\partial_{\epsilon} f_{i}(0)=\varnothing$. Therefore, formula (3.39) does not hold for $x=0$ while $\{0\}=\operatorname{dom} \psi$ (and (3.37) fails).

Example 3.2. Let $X=\mathbb{R}^{2}$ and $I=\{1,2\}$. Define the sets $A_{1}$ and $A_{2}$, respectively, by

$$
A_{1}:=\left\{\left(t_{1}, t_{2}\right) \in \mathbb{R}^{2}: t_{2}<0\right\} \cup\{(0,0)\} \quad \text { and } \quad A_{2}:=\left\{\left(t_{1}, t_{2}\right) \in \mathbb{R}^{2}: t_{2}>0\right\} \cup\{(0,0)\} \text {. }
$$

Consider the family $\left\{f_{i}: i \in I\right\}$ with each $f_{i}:=\delta_{A_{i}}$. Then $\operatorname{cl} f_{i}=\delta_{\operatorname{cl} A_{i}}$ for each $i \in I$ and $\psi:=\sup _{i \in I} f_{i}=\delta_{\{0\}}$. Therefore, $\operatorname{cl}(\operatorname{dom} \psi)=\{0\}$ and (3.37) is satisfied by the family $\left\{f_{i}: i \in I\right\}$ but not condition (1.1) (which is a part of assumptions in [11, Theorem 4]). Thus Corollary 3.2 is applicable but not [11, Theorem 4].

4. Conclusions. We have established, by using the powerful epigraph technique, the subdifferential formula for the supremum function $\psi$ at $z \in \operatorname{dom} \psi$ in terms of the $\epsilon$-subdifferentials of the data functions $f_{i}$ at $z$. In particular, our Corollary 3.2 extends and improves the latest results due to Hantoute, López, and Zălinescu in [11]. It is tempting to prove Corollary 3.2 by virtue of the corresponding known results and via the following approach.

Define a new family $\left\{\hat{f}_{i}: i \in I\right\}$ of proper convex functions on $X$ by

$$
\hat{f}_{i}:=f_{i}+\delta_{\mathrm{cl}(\operatorname{dom} \psi)} \quad \text { for each } i \in I .
$$

We use $\hat{\psi}$ to denote the corresponding sup-function, that is, $\hat{\psi}=\sup _{i \in I} \hat{f}_{i}$. Then it is clear that

$$
\hat{\psi}=\sup _{i \in I} f_{i}+\delta_{\mathrm{cl}(\operatorname{dom} \psi)}=\psi+\delta_{\mathrm{cl}(\operatorname{dom} \psi)}=\psi
$$

Moreover, by definition, we can show directly that condition (3.37) implies the following one:

$$
\operatorname{cl} \hat{\psi}=\sup _{i \in I}\left(\operatorname{cl} \hat{f}_{i}\right)
$$

Thus, under the assumption of Corollary 3.2, we can apply [11, Theorem 4] (to $\left.\left\{\hat{f}_{i}: i \in I\right\}\right)$ to obtain that 


$$
\partial \psi(x)=\underset{\substack{\epsilon>0 \\ L \in \mathcal{F}_{x, \mathrm{cl}(\operatorname{dom} \psi)}}}{\cap} \operatorname{cl}\left(\operatorname{co} \cup_{i \in I_{\epsilon}(x)} \partial_{\eta \epsilon}\left(f_{i}+\delta_{\mathrm{cl}(\operatorname{dom} \psi)}\right)(x)+\mathrm{N}_{L \cap \operatorname{dom} \psi}(x)\right) .
$$

To proceed further, we have to calculate the $\epsilon$-subdifferential of $f_{i}+\delta_{\mathrm{cl}(\mathrm{dom} \psi)}$ at $x$ for each $i \in I_{\epsilon}(x)$. To the best of our knowledge, the known formulas for the $\epsilon$-subdifferential of the sum of two proper convex functions $f$ and $g$ (see [29, Corollary 2.6.7]) require that the involved functions $f$ and $g$ are lsc. They can probably be extended to the case when the involved two functions $f$ and $g$ have the property

$$
\operatorname{cl}(f+g)=\operatorname{cl} f+\operatorname{cl} g
$$

even so, they cannot be applied in our situation here because, in general, condition (3.37) does not imply that (4.2) holds with $f:=f_{i}$ and $g:=\delta_{\mathrm{cl}(\operatorname{dom} \psi)}$ even for $i \in I_{\epsilon}(x)$ as shown by the following example of the family $\left\{f_{i}: i \in I\right\}$. Therefore, we guess, the approach outlined above does not work for deriving Corollary 3.2.

Example 4.1. Let $X:=\mathbb{R}^{2}$ and $I:=(0,1]$. Consider the family $\left\{f_{i}: i \in I\right\}$ consisting of proper convex functions $f_{i}$ defined by

$$
f_{i}(x):= \begin{cases}0, & x \in[0,1] \times\{0\}, \\ -i, & x \in[0,1] \times(0, i], \quad \text { for each } i \in I . \\ +\infty, & \text { otherwise }\end{cases}
$$

Then we have that $\psi=\sup _{i \in I} f_{i}=\delta_{[0,1] \times\{0\}}$ and

$$
\operatorname{cl} f_{i}=-i+\delta_{[0,1] \times[0, i]} \text { for each } i \in I .
$$

This means that $\operatorname{cl}(\operatorname{dom} \psi)=[0,1] \times\{0\}$ and condition (3.37) is satisfied. Moreover, for any $x \in[0,1] \times\{0\}$ and any $\epsilon>0$, one sees that $I_{\epsilon}(x)=I$ and that, for each $i \in I$,

$$
\operatorname{cl}\left(f_{i}+\delta_{\mathrm{cl}(\operatorname{dom} \psi)}\right)=\delta_{\mathrm{cl}(\operatorname{dom} \psi)}>-i+\delta_{\mathrm{cl}(\operatorname{dom} \psi)}=\operatorname{cl} f_{i}+\delta_{\mathrm{cl}(\operatorname{dom} \psi)} \quad \text { on }[0,1] \times\{0\} .
$$

Hence (4.2) does not hold with $f:=f_{i}$ and $g:=\delta_{\operatorname{cl}(\operatorname{dom} \psi)}$ for each $i \in I_{\epsilon}(x)$.

Acknowledgments. The authors are indebted to two anonymous referees for their helpful remarks, which allowed us to improve the original presentation.

\section{REFERENCES}

[1] J. Borwein and R. Goebel, Notions of relative interior in Banach spaces, J. Math. Sci., 115 (2003), pp. 2542-2553.

[2] A. BRøndsted, On the subdifferential of the supremum of two convex functions, Math. Scand., 31 (1972), pp. 225-230.

[3] J. M. Danskin, The Theory of Max-Min and its Application to Weapons Allocation Problems, Springer, Berlin, 1967.

[4] D. H. FAnG, C. Li, AND K. F. NG, Constraint qualifications for extended Farkas's lemmas and Lagrangian dualities in convex infinite programming, SIAM J. Optim., 20 (2009), pp. 1311-1332.

[5] D. H. FANG, C. LI, AND K. F. NG, Constraint qualifications for optimality conditions and total Lagrange dualities in convex infinite programming, Nonlinear Anal., 73 (2010), pp. 1143-1159.

[6] N. Dinh, M. A. Goberna, and M. A. López, From linear to convex systems: Consistency, Farkas' lemma and applications, J. Convex Anal., 13 (2006), pp. 113-133.

[7] N. Dinh, M. A. Goberna, M. A. López, and T. Q. Son, New Farkas-type constraint qualification in convex semi-infinite programming, ESAIM Control Optim. Calc. Var., 13 (2007), pp. 580-597.

Copyright @ ( by SIAM. Unauthorized reproduction of this article is prohibited. 
[8] M. A. Goberna and M. A. López, Linear Semi-Infinite Optimization, Wiley Ser. Math. Methods Pract., John Wiley and Sons, Chichester, 1998.

[9] A. Hantoute, Subdifferential set of the supremum of lower semicontinuous convex functions and the conical hull intersection property, TOP, 14 (2006), pp. 355-374.

[10] A. Hantoute and M. A. López, A complete characterization of the subdifferential set of the supremum of an arbitrary family of convex functions, J. Convex Anal., 15 (2008), pp. 831-858.

[11] A. Hantoute, M. A. López, And C. ZăLinescu, Subdifferential calculus rules in convex analysis: A unifying approach via pointwise supremum functions, SIAM J. Optim., 19 (2008), pp. 863-882.

[12] J. Hiriart-Urruty and C. Lemarechal, Convex Analysis and Minimization Algorithms I, Grundlehren Math. Wiss. 305, Springer-Verlag, Berlin, 1993.

[13] J.-B. Hiriart-Urruty, M. Moussaoui, A. Seeger, and M. Volle, Subdifferential calculus without qualification conditions, using approximate subdifferentials: A survey, Nonlinear Anal., 24 (1995), pp. $1727-1754$.

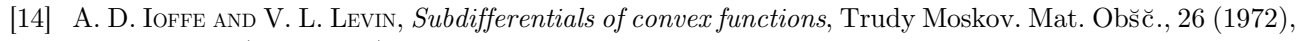
pp. 3-73 (in Russian).

[15] A. D. Ioffe and V. H. Tiknomirov, Theory of Extremal Problems, in Stud. Math. Appl. 6, North-Holland, Amsterdam, 1979.

[16] V. Jeyakumar, A. M. Rubinov, B. M. Glover, and Y. Ishizuka, Inequality systems and global optimization, J. Math. Anal. Appl., 202 (1996), pp. 900-919.

[17] V. L. Levin, An application of Hellys theorem in convex programming, problems of best approximation and related questions, Mat. Sb., 79 (1969), pp. 250-263 (in Russian); Sb. Math., 8 (1969), pp. 235-247 (in English).

[18] C. Li and K. F. NG, On constraint qualification for an infinite system of convex inequalities in a Banach space, SIAM J. Optim., 15 (2005), pp. 488-512.

[19] C. Li, K. F. NG, ANd T. K. Pong, Constraint qualifications for convex inequality systems with applications in constrained optimization, SIAM J. Optim., 19 (2008), pp. 163-187.

[20] W. Li, C. NAHAK, AND I. Singer, Constraint qualifications for semi-infinite systems of convex inequalities, SIAM J. Optim., 11 (2000), pp. 31-52.

[21] B. N. Pschenichnyi, Convex programming in a normalized space, Kibernetika, 5 (1965), pp. $46-54$ (in Russian); Cybernetics, 1 (1965), pp. 46-57 (in English).

[22] R. T. Rockafellar, Directionally Lipschitzian functions and subdifferential calculus, Proc. London Math. Soc. (3), 39 (1979), pp. 331-355.

[23] V. N. Solovev, The subdifferential and the directional derivatives of the maximum of a family of convex functions, Izv. Ross. Akad. Nauk Ser. Mat., 65 (2001), pp. 107-132 (in Russian); Izv. Math., 65 (2001), pp. 99-121 (in English).

[24] L. Thibault, Sequential convex subdifferential calculus and sequential Lagrange multipliers, SIAM J. Control Optim., 35 (1997), pp. 1434-1444.

[25] V. M. Tikнomirov, Analysis II, Convex Analysis and Approximation Theory, Encyclopedia Math. Sci. 14, R. V. Gamkrelidze, ed., Springer-Verlag, New York, 1987.

[26] M. Valadier, Sous-différentiels d'une borne supérieure et d'une somme continue de fonctions convexes, C. R. Acad. Sci. Paris Sér. A-B Math., 268 (1969), pp. A39-A42.

[27] M. Volle, Sous-différentiels d'une enveloppe supérieure de fonctions convexes, C. R. Acad. Sci. Paris Sér. I Math., 317 (1993), pp. 845-849.

[28] M. VolLe, On the subdifferential of an upper envelope of convex functions, Acta Math. Vietnam., 19 (1994), pp. 137-148.

[29] C. Zalinescu, Convex Analysis in General Vector Spaces, World Scientific, Singapore, 2002.

Copyright (C) by SIAM. Unauthorized reproduction of this article is prohibited. 